\title{
Autologous fat transfer after breast cancer surgery: an exact-matching study on the long-term oncological safety
}

Luca Sorrentino ${ }^{\mathrm{a}}, \mathrm{MD}$, Lea Regolo ${ }^{\mathrm{b}}$, MD, Elisabetta Scoccia ${ }^{\mathrm{b}}$, MD, Gianfranco Petrolo ${ }^{\mathrm{b}}, \mathrm{MD}$, Daniela Bossi ${ }^{\mathrm{b}}$, MD, Sara Albasini ${ }^{\mathrm{b}}$, MSc, Annalisa Caruso ${ }^{\mathrm{a}}, \mathrm{MD}$, Renzo Vanna ${ }^{\mathrm{c}}, \mathrm{PhD}$, Carlo Morasso $^{\mathrm{c}}, \mathrm{PhD}$, Serena Mazzucchelli ${ }^{\mathrm{a}}, \mathrm{PhD}$, Marta Truffi ${ }^{\mathrm{a}}, \mathrm{PhD}$, Fabio Corsi ${ }^{\mathrm{a}, b^{*}}, \mathrm{MD}$

aDepartment of Biomedical and Clinical Sciences “Luigi Sacco", University of Milan, via G. B. Grassi 74, 20157 Milan, Italy

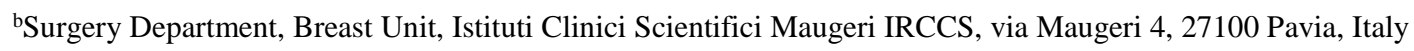

${ }^{\mathrm{c}}$ Nanomedicine and Molecular Imaging Lab, Istituti Clinici Scientifici Maugeri IRCCS, via Maugeri 4, 27100 Pavia, Italy

*Corresponding Authors:

Prof. Fabio Corsi, MD

Department of Biomedical and Clinical Sciences "Luigi Sacco”, University of Milan, Via G. B. Grassi, 74, 20157 Milan, Italy

Surgery Department, Breast Unit, ICS Maugeri S.p.A. SB, via Maugeri 10, 27100 Pavia, Italy

E-mail: fabio.corsi@ unimi.it - Phone: +39.02.5031.9850 - Fax: +39.02.5031.9846

Declarations of interest: None.

Role of the funding source: None.

Funding: This research did not receive any specific grant from funding agencies in the public, commercial, or not-for-profit sectors. 


\begin{abstract}
Introduction: Autologous fat transfer (AFT) is widely adopted for breast reconstruction, but its longterm oncologic safety is still not clearly established. The aim of the present study was to compare the 10-year loco-regional recurrence (LRR)-free and distant metastases (DM)-free survival probabilities in AFT vs. control patients, also evaluating the impact of AFT in different intrinsic molecular subtypes of breast cancer.
\end{abstract}

Materials and Methods: 464 AFT patients were exactly matched with a cohort of 3,100 control patients treated between 2007 and 2017. A multivariate survival analysis was performed accounting for all variables related to LRR and DM, including adjuvant/neoadjuvant treatments. End-points were analyzed both overall and in each molecular subtype.

Results: LRR occurred in $6.4 \%$ of AFT and in $5.0 \%$ of control patients $(\mathrm{p}=0.42)$, while DM were observed respectively in $7.7 \%$ and $5.4 \%$ of cases $(\mathrm{p}=0.20)$. AFT showed no effect on the 10 -year LRR-free survival probability (adjusted HR 0.87, 95\%CI 0.43-1.76, $\mathrm{p}=0.69$ ) or the 10-year DM-free survival probability (adjusted HR $0.82,95 \%$ CI $0.43-1.57, \mathrm{p}=0.55$ ). Luminal A patients treated by AFT showed a decreased LRR-free survival probability (HR 2.38, 95\%CI 0.91-6.17, Log-Rank $\mathrm{p}=0.07$ ), which was significantly lower than controls after 80 months (Log-Rank $\mathrm{p}=0.02)$. No differences in the 10-year event-free survival probability were found in Luminal B, HER2-positive or triple-negative patients.

Conclusion: AFT does not increase breast cancer recurrence, with the possible exception of late LRRs for Luminal A patients, but further clinical and preclinical data are required to better clarify this data. The use of AFT should not be discouraged.

\title{
Keywords
}

Autologous fat transfer; Lipofilling; Breast cancer; Loco-regional recurrence; Distant metastasis 


\section{Introduction}

Autologous fat transfer (AFT) consists in adipose fat tissue liposuction from donor sites, its purification and its relocation to breast sites, to refill a volume defect or to correct other asymmetries or irregularities after breast-conserving surgery or mastectomy [1, 2]. This technique is widely adopted by breast surgeons, and its use has risen over time [3]. However, concerns about an increased risk of cancer recurrence have been raised, and the oncological safety of AFT has been questioned [4-7]. Indeed, adipose-derived mesenchymal stem cells are transferred together with adipose tissue during AFT, stimulating angiogenesis and stroma remodeling, thus being responsible for the cosmetic success of lipofilling [8,9]. On the other hand, preclinical studies showed that adipocytes and adipokines, being part of the tumor microenvironment, may play a role in cancer occurrence and progression, possibly being related to recurrence [10-13]. A few case-control studies based on large populations are available today, which suggest that local recurrence rates do not seem increase after AFT $[7,14]$. At the same time, rigorous evidence analyzing long-term oncologic outcomes and which also considers adjuvant treatments and biomolecular subtypes (BMS) of breast cancer are still scarce [14]. The aim of the present study was to assess the long-term oncologic impact of AFT, by comparing the 10-year loco-regional and distant recurrence-free survival probability in patients treated for breast cancer with or without AFT, also evaluating the correlation with BMS and adjuvant therapies in a long-term follow up. 


\section{Material and methods}

\subsection{Study population}

Patients were retrospectively collected from the prospectively-maintained database of the EUSOMAaccredited Breast Unit of ICS Maugeri Hospital from January 2007 to December 2017. The 464 patients who received at least one AFT were selected and exactly matched with a cohort of control patients extracted from the remaining 3,100 women who were not subjected to AFT. Inclusion criteria were: proven diagnosis of breast cancer and any surgery including lumpectomy, nipple-sparing mastectomy or skin-sparing mastectomy with reconstruction. Patients with benign diseases, distant metastases at diagnosis, a previous diagnosis of breast cancer or other cancer, or with relevant data missing were excluded from the study.

\subsection{Study design and endpoints}

Due to the non-random design of the study, an exact match of categorical variables and a range of values for continuous variables were designated, matching patients treated with AFT and control patients according to the subsequent variables: age, histopathology, staging, BMS and grading. BMS were approximated as follows, based on the histopathological features of breast cancer: luminal A (positive hormone receptors and negative HER2, Ki67 <14\%), luminal B (positive hormone receptors and any HER2 status, Ki67 $\geq 14 \%$ ), HER2-enriched (negative hormone receptors and positive HER2), and triple-negative (negative hormone receptors and negative HER2). AFT patients and controls were matched allowing a 1:4 ratio at most. To provide a reliable comparison between AFT and control patients in terms of follow-up, each selected control had an event-free follow-up for at least as long as the period between breast cancer surgery and the AFT of the matched case. The main endpoints were 1) the 10-year loco-regional recurrence (LRR)-free survival probability and 2) the 10-year distant metastases (DM)-free survival probability in patients treated with AFT vs. controls. LRR was defined as the occurrence of ipsilateral breast cancer or nodal disease at axillary, internal mammary and/or supraclavicular level, proven on core biopsy. DM was defined as the occurrence of distant 
lesions with computed tomography and positron emission tomography features suggestive of malignancy, even if not biopsy-proved. A multivariate survival analysis was performed accounting for all variables related to LRR and DM, including adjuvant or neoadjuvant treatments and BMS. Secondary endpoints were: 1) cancer-related death rate, 2) impact of AFT on LRR and DM stratified by number of AFT procedures, time between breast cancer surgery and AFT, and type of surgery, and 3) evaluation of long-term oncologic safety of AFT according to each BMS.

\subsection{AFT technique}

Indications for AFT were: contour defects, asymmetry, scar retraction and fibrosis, or volume defect after any type of breast cancer surgery; thinning of peri-prosthetic tissues after mastectomy and breast reconstruction, particularly in the case of increased risk of implant exposure; capsular contracture after implant reconstruction. Preferred donor sites were abdominal subcutaneous tissue or fatty tissue from thighs. The chosen area was infiltrated with Klein's solution (average $200 \mathrm{~mL}$ ) prepared as follows: $60 \mathrm{mg}$ of lidocaine, $7.5 \mathrm{mg}$ of ropivacaine and $1 \mathrm{mg}$ of adrenaline mixed in $1 \mathrm{~L}$ of cold saline solution at $0.9 \%$. Infiltration was performed through the subdermal tissue of the donor site using an $18 \mathrm{G}$ needle connected to a $50 \mathrm{~mL}$ syringe. After infiltration, a $5 \mathrm{~mm}$ skin incision was performed on the donor site and a blunt-tipped Coleman cannula with a diameter of $4 \mathrm{~mm}$ was inserted. Liposuction by negative pressure was then started. The aspirated adipose tissue was then centrifuged (IEC Medispin-6 Krackeler Scientific Inc., Albany, NY) at 2700xg for 3-4 minutes to separate adipocytes from erythrocytes, lysed cells and serum. The purified fat tissue was then collected and slowly injected into the subdermal space of the receiving breast site.

\subsection{Statistical analysis}

Differences between AFT and control patients after exact matching were assessed to verify the heterogeneity of the study population. Variables were reported as mean \pm standard deviations or as absolute numbers and percentages. Categorical variables were compared using a $\chi^{2}$ test or Fisher 
exact test when the sample size was less than or equal to 5, while continuous variables were compared using a Student's T test or non-parametric Wilcoxon test in case of the variable's non-normal distribution. AFT and control patients were compared by a Cox proportional hazards regression model, including variables significantly associated with the outcomes, such as the type of adjuvant/neoadjuvant therapy (hormone therapy, chemotherapy and radiation therapy) and BMS to avoid any bias. The 10-year LRR-free and DM-free survival probabilities were estimated by the Kaplan-Meier method, both globally and in each BMS. Statistical significance was set at $\mathrm{p}<0.05$ (twotailed). Data analysis was performed using SAS software (v. 9.4, SAS Institute Inc., Cary, USA).

\section{Results}

3.1 Distribution of baseline variables and type of adjuvant treatments after exact matching 
After exact matching, 830 patients were included for outcomes analyses: 233 AFT (28.1\%) and 597 control patients $(71.9 \%)$. The distribution of baseline variables and BMS were balanced between groups, as reported in Table 1 . AFT patients were treated more frequently by mastectomy when compared to controls ( $76.8 \%$ vs. $25.6 \%, \mathrm{p}<0.0001)$. Consequently, control patients were more likely to receive radiation therapy when compared to AFT cases $(74.9 \%$ vs. $45.9 \%, \mathrm{p}<0.0001)$. In particular, whole breast radiotherapy with or without boost was offered to $45.8 \%$ of AFT patients and to $53.6 \%$ of the control group, thoracic wall and/or loco-regional radiotherapy were performed respectively in $38.3 \%$ and $25.4 \%$ of cases, and other types of radiotherapy were delivered in $15.9 \%$ and $21.0 \%$ of patients $(\mathrm{p}=0.053)$, Table 1 . Chemotherapy was administered in $54.1 \%$ of AFT patients vs. $44.6 \%$ of control patients $(\mathrm{p}=0.04)$; neoadjuvant treatment was proposed respectively in $12.0 \%$ and $8.4 \%$ of cases (Table 1). Chemotherapy regimen was based on anthracyclines with or without taxanes in $64.7 \%$ of AFT vs. $57.1 \%$ of control patients, and trastuzumab was administered respectively in $26.1 \%$ and $34.8 \%$ of cases; in the remaining cases, cyclophosphamide with methotrexate and fluorouracil $(5.9 \%$ vs. $5.0 \%)$ or other regimens $(3.4 \%$ vs. $3.1 \%)$ were offered $(\mathrm{p}=0.48)$.

\subsection{Long-term outcomes and event-free survival probabilities between AFT vs. no AFT patients}

Mean follow-up after breast cancer surgery was $74.1( \pm 40.4)$ months in AFT patients vs. $63.8( \pm 37.1)$ months in controls ( $\mathrm{p}=0.0015)$. In the AFT group, the mean time interval from breast cancer surgery to the first lipofilling procedure was $22.9( \pm 19.7)$ months, matched with an event-free interval which was at least equal in control patients. The, mean follow-up period after the first lipofilling procedure was $51.2( \pm 35.0)$ months. LRR occurred in $6.4 \%$ of AFT patients and in $5.0 \%$ of control patients $(p=0.42)$, while DM were observed respectively in $7.7 \%$ and $5.4 \%$ of cases $(p=0.20)$. Furthermore, no difference was observed in cancer-related death rates, being $5.1 \%$ in AFT vs. $3.4 \%$ in controls $(\mathrm{p}=0.23)$. All these data are reported in Table 2. AFT and control patients showed comparable 10year LRR-free survival probability curves, with a hazard ratio (HR) for AFT vs. no AFT equal to 0.96 (95\%CI 0.52-1.80, Log-Rank test $\mathrm{p}=0.91)$, see Figure 1a. Also, 10-year DM-free survival 
probabilities were similar between AFT and control patients (HR 1.24, 95\%CI 0.69-2.21, Log-Rank test $\mathrm{p}=0.47$ ), as shown in Figure $1 \mathrm{~b}$.

\subsection{Multivariate survival analysis of AFT vs. no AFT on LRR-free and DM-free survival probabilities} After adjusting for potential confounding variables by means of the Cox regression model, AFT confirmed no effect on 10-year LRR-free survival probability, with a HR equal to 0.87 (95\%CI 0.43$1.76, \mathrm{p}=0.69)$. No variable resulted to be independently associated with LRR. AFT was not associated with decreased 10-year DM-free survival probability (HR 0.82, 95\%CI 0.43-1.57, $\mathrm{p}=0.55)$. The only factor independently associated with DM was the need for adjuvant (HR 2.81, 95\% CI 1.10-7.14, $\mathrm{p}=0.03$ ) or neoadjuvant (HR 5.63,95\%CI 2.01-15.75, $\mathrm{p}=0.001)$ chemotherapy. Menopausal status and body mass index showed no significant association with LRR or DM. The Cox multivariate analysis is reported in Table 3.

3.4 Impact of number of AFT procedures, time between oncologic surgery and AFT and type of surgery on outcomes

Taking all AFT patients ( $\mathrm{n}=464)$ into consideration, no correlation was found between the number of lipofilling procedures and the occurrence of LRR or DM (respectively Log-rank $p=0.28$ and $p=0.45$ ), as reported in Table 4. Performing AFT early ( $<1$ year from oncologic surgery) was not associated with increased events (for LRR Log-rank $\mathrm{p}=0.44$, for DM Log-rank $\mathrm{p}=0.74$ ), despite a higher proportion of early AFT observed among recurrent patients (36.0\% vs. $24.2 \%)$. Use of AFT after breast-conserving surgery, nipple-sparing mastectomy or skin-sparing mastectomy was not related to LRR (Log-rank p=0.85) or DM (Log-rank p=0.33).

\subsection{Impact of AFT on outcomes in different BMS of breast cancer}

A sub-analysis was performed comparing the effect of AFT vs. no AFT on 10-year LRR-free and DM-free survival probabilities in different BMS of breast cancer (Figure 2). AFT was not associated 
with increased frequency of LRR (HR 2.38, 95\%CI 0.91-6.17, Log-Rank p=0.07) and DM (HR 2.18, 95\%CI 0.94-5.06, Log-Rank p=0.06) in Luminal A patients, considering the overall follow-up. However, starting from 80 months, survival probability curves were different between the remaining AFT patients $(n=45)$ and controls $(n=100)$ for LRR (Log-Rank $p=0.02$, Figure 2a) but not for DM (Log-Rank p=0.06, Figure 2b). No differences were observed for Luminal B (LRR: HR 0.51, 95\%CI 0.16-1.60, Log-Rank p=0.24, Figure 2c; DM: HR 1.05, 95\%CI 0.39-2.81, Log-Rank p=0.91, Figure 2d) and HER2-positive breast cancers (LRR: HR 1.97, 95\%CI 0.33-11.89, Log-Rank p=0.45, Figure 2e; DM: HR 0.79, 95\%CI 0.08-7.76, Log-Rank p=0.84, Figure 2f). Also, no differences were found in triple-negative breast cancers, where no events were encountered among AFT patients (LRR: LogRank $\mathrm{p}=0.07$, Figure 2g; DM: Log-Rank $\mathrm{p}=0.12$, Figure 2h).

\section{Discussion}


At least 41 non-overlapping studies have previously reported LRR and DM rates after AFT, but the oncologic safety of lipofilling still remains debated, despite the fact that the great majority of evidence suggests similar outcomes between AFT and control patients [7]. We observed that LRR and DM rates were similar between AFT and control patients after exact matching according to baseline cancer-related features. After adjusting for confounders in a regression model accounting for adjuvant treatments and BMS, AFT was still not associated with increased LRR (HR 0.87, p=0.69) or DM (HR 0.82, $\mathrm{p}=0.55$ ). Recently, a large meta-analysis on 4,292 patients demonstrated a non-significant incidence rate difference in LRR of $-0.15 \%$ per year between AFT and control patients, providing robust evidence of AFT safety after breast cancer [7]. However, the mean follow-up from AFT procedure was only 2.7 years for included studies. Considering the long-lasting natural history of breast cancer, such a short follow-up is a common concern due to the risk of under-estimation in cumulative incidence of events $[15,16]$. In the present study, a longer follow-up is available for AFT patients, with a mean of 51.2 months after the first lipofilling procedure. As can be expected, a longer overall follow-up was available for AFT patients (74.1 vs. 63.8 months, $\mathrm{p}=0.0015)$, since AFT is generally reserved for patients after a certain disease-free interval. To better control for this bias, we matched control patients with a disease-free period at least equal to the period from breast cancer surgery to the first lipofilling in AFT patients. This solution was first proposed by Petit and colleagues in their landmark study on 321 AFT patients, providing a reliable comparison which showed no impact of AFT on LRR (HR 1.11, p=0.792) [17]. Also, Krastev et al. reported a longer overall followup for AFT patients compared to control, being respectively 9.3 vs. 8.6 years but again, the LRR-free interval of control patients was matched with the period between breast cancer surgery and exposure to AFT [15]. No effect of AFT on LRR was found, with a HR of 0.63 ( $p=0.33)$, confirming that evidence of higher risk after AFT is lacking.

A major point of concern is the unbalanced proportion of patients who underwent mastectomy vs. breast-conserving surgery in the two groups, with mastectomy performed in $76.8 \%$ of AFT patients 
vs. only $25.6 \%$ of controls $(\mathrm{p}<0.0001)$ after exact matching. Since AFT can correct soft tissue deformities, skin flap thinning, capsular contracture and post-mastectomy pain syndrome [18, 19], it is not surprising that AFT was adopted after mastectomy in the great majority of patients in this study and in previous literature $[7,16,20-22]$. Due to the different distribution of types of surgery in the two groups, AFT patients received significantly less radiotherapy when compared to controls (45.9\% vs. 74.9\%, p<0.0001). Apparently, such differences could represent a bias for our findings. However, it is currently well-established that lumpectomy with radiotherapy carries a similar LRR risk when compared to mastectomy $[23,24]$, and that biological features of breast cancer together with adjuvant treatments predict LRR better than the extent of surgery [25]. Therefore, the difference in the type of surgery is conceptually irrelevant in terms of the LRR rate, considering that the clinical, pathological and biological characteristics of patients were exactly matched and taken into account in the multivariate analysis. Furthermore, the type of surgery was in any case included in the multivariate survival analysis together with the use of AFT, and it confirmed that there was no association with LRR (HR 0.84, p=0.71) or DM (HR 1.09, p=0.84).

In our institution, loco-regional irradiation is proposed only in patients with $>3$ positive nodes irrespective of the type of surgery, thus avoiding post-mastectomy radiotherapy in N1 patients. Since the stage was similar between groups, no significant differences were found in the type of irradiation, being loco-regional radiotherapy offered in $38.3 \%$ of AFT patients vs. $25.4 \%$ of controls ( $\mathrm{p}=0.053$ ). Moreover, the use of radiotherapy was considered in multivariate analysis, but it was not related to LRR (HR 1.04, p=0.93) or DM (HR 0.71, p=0.40). Consequently, it is unlikely that loco-regional irradiation in AFT patients could have biased survival analyses.

Indeed, some studies suggest that in AFT after breast-conserving surgery, adipose tissue may crosstalk with surrounding dormant residual cancer cells, thus promoting LRR [26, 27]. In this study, the type of surgery was equally distributed among AFT patients with and without LRR ( $\mathrm{p}=0.72)$, and 
breast-conserving surgery was performed respectively in $26.4 \%$ and $20.0 \%$ of cases. This further excludes the role of the type of surgery in LRR and confirms the safety of AFT after breast-conserving surgery. The largest population of patients undergoing breast-conserving surgery followed by AFT comes from a recent study by Petit, which confirmed that AFT is a safe procedure even after lumpectomy [28]. Petit and Silva-Vergara suggested that AFT performed within 3 years after breast cancer surgery is related to increased risk of LRR $[22,26]$. In the present study, the great majority of patients (about 70\%) received the first AFT procedure within 3 years from oncologic surgery, but this has not resulted in an increased event rate $(\mathrm{p}=0.44$ for LRR and $\mathrm{p}=0.74$ for $\mathrm{DM})$.

No study is known to have specifically addressed the risk of AFT on LRR or DM in relation to fundamental predictors of outcome such as BMS $[7,15-17,22,26]$. Luminal cancers are related to late recurrences and HER2-positive or triple-negative cancers to early events [29-32]. Considering the global follow-up of Luminal A, no significant decrease in LRR-free (HR 2.38, p=0.07) or DMfree (HR 2.18, $\mathrm{p}=0.06$ ) survival probabilities was observed in AFT patients. However, after 80 months a significant trend toward a lower LRR-free survival probability seems to be observed in AFT patients affected by Luminal A cancers ( $\mathrm{p}=0.02$ ). Despite this intriguing finding, it should be noted that after 80 months only 145 Luminal A patients were still on follow-up, representing less than onethird of the initial Luminal A population. Therefore, no definitive conclusions on the impact of AFT on the Luminal A subtype may be drawn. There is no previous study which reports this effect of AFT on late LRR in Luminal A cancers, and this could be related to the shorter mean follow-up available in other studies, which could lead to missing late LRR typically associated with Luminal cancers [7]. Kronowitz et al. demonstrated that AFT patients undergoing hormone therapy had a three-fold risk of LRR when compared to controls [16]. The authors suggested that hormone-related pathways could somehow crosstalk with adipose-derived mesenchymal stem cells promoting recurrence, but no clear explanation could be offered. Also Petit described that LRRs significantly increased after AFT in DCIS [26]. Notably, $80 \%$ of tumors were estrogen receptor-positive. Therefore, in both Kronowitz 
and Petit studies, it is not clear whether the different outcome of AFT reported in those patient subsets might be better related to a Luminal-type BMS. Another finding of the present study is the unexpectedly low rate of LRR among lipofilled triple-negative patients $(\mathrm{p}=0.07)$. This data could be explained by the fact that triple-negative breast cancer typically recurs with early events, thus excluding the indication for AFT [31]. Therefore, triple-negative AFT patients were mostly represented by cases with a longer disease-free survival compared to controls.

\section{Study limitations}

This study presents some limitations. First, it is not a randomized clinical trial therefore, patients were retrospectively allocated into two groups (AFT vs. no AFT) potentially associated with different LRR and DM risks. Indeed, AFT is often performed on young patients undergoing mastectomy for locallyadvanced tumors at presentation, with higher T stages and more frequent axillary involvement [33]. This main difference accounts for the unbalanced distribution of variables observed in Table 1, particularly the overwhelming use of mastectomy in the AFT group, despite the matching of preoperative variables. However, exact matching and subsequent multivariate analysis were performed to properly address these biases. Second, radiotherapy was used differently between groups and loco-regional irradiation was favored slightly in AFT patients. Therefore, radiotherapy's possible role in biasing LRR analysis may remain unclear. Third, the exact matching of AFT and control patients greatly reduced the study population, thus flawing the study's statistical power. In particular, data on the impact of AFT on Luminal A patients after 80 months might not be robust enough to support relevant conclusions.

\section{Conclusions}

AFT could be safely proposed in the setting of multi-modal adjuvant treatment. A possible increase in late LRRs for Luminal A patients undergoing AFT cannot be excluded however, further clinical and preclinical data are required to better elucidate this point. 


\section{Conflict of Interest Statement}

None declared.

\section{References}

1. Krastev TK, Alshaikh GAH, Hommes J, Piatkowski A, van der Hulst RRWJ. Efficacy of autologous fat transfer for the correction of contour deformities in the breast: A systematic review and meta-analysis. J Plast Reconstr Aesthet Surg 2018; 71(10):1392-1409. 
2. Missana MC, Laurent I, Barreau L, Balleyguier C. Autologous fat transfer in reconstructive breast surgery: indications, technique and results. Eur J Surg Oncol 2007; 33(6):685-90.

3. Groen JW, Piatkowski AA, Sawor JH, Wilschut JA, Ritt MJPF, van der Hulst RRJW. European Survey Study Among Plastic/Breast Surgeons on the Use of and Opinion Toward Autologous Fat Transfer: With Emphasis on Breast Surgery. Surg Innov 2018; in press.

4. Bertolini F, Petit JY, Kolonin MG. Stem cells from adipose tissue and breast cancer: hype, risks and hope. Br J Cancer 2015; 112(3):419-23.

5. Lohsiriwat V, Curigliano G, Rietjens M, Goldhirsch A, Petit JY. Autologous fat transplantation in patients with breast cancer: "silencing" or "fueling" cancer recurrence? Breast 2011; 20(4):351-7.

6. Petit JY, Clough K, Sarfati I, Lohsiriwat V, de Lorenzi F, Rietjens M. Lipofilling in breast cancer patients: from surgical technique to oncologic point of view. Plast Reconstr Surg 2010; 126(5):262e-263e.

7. Krastev TK, Schop SJ, Hommes J, Piatkowski AA, Heuts EM, van der Hulst RRWJ. Metaanalysis of the oncological safety of autologous fat transfer after breast cancer. Br J Surg 2018; 105(9):1082-1097.

8. Brown SA, Levi B, Lequeux C, Wong VW, Mojallal A, Longaker MT. Basic science review on adipose tissue for clinicians. Plast Reconstr Surg 2010; 126(6):193646.

9. Rehman J, Traktuev D, Li J, Merfeld-Clauss S, Temm-Grove CJ, Bovenkerk JE, et al. Secretion of angiogenic and antiapoptotic factors by human adipose stromal cells. Circulation 2004; 109(10):1292-8.

10. Jablonka A, Scheich J, Jacobsen F, Hirsch T, Hagouan M, Lehnhardt M, et al. Influence of preadipocyte-conditioned medium on the proliferation and invasive potential of breast cancer cell lines in vitro. Arch Gynecol Obstet 2018; 298(6):1159-1171. 
11. Choi J, Cha YJ, Koo JS. Adipocyte biology in breast cancer: From silent bystander to active facilitator. Prog Lipid Res 2018; 69:11-20.

12. Kuhbier JW, Bucan V, Reimers K, Strauss S, Lazaridis A, Jahn S, et al. Observed changes in the morphology and phenotype of breast cancer cells in direct co-culture with adipose-derived stem cells. Plast Reconstr Surg. 2014; 134(3):414-23.

13. Wang YY, Lehuédé C, Laurent V, Dirat B, Dauvillier S, Bochet L, et al. Adipose tissue and breast epithelial cells: a dangerous dynamic duo in breast cancer. Cancer Lett 2012; 324(2):142-51.

14. Waked K, Colle J, Doornaert M, Cocquyt V, Blondeel P. Systematic review: The oncological safety of adipose fat transfer after breast cancer surgery. Breast 2017; 31:128-136.

15. Krastev T, van Turnhout A, Vriens E, Smits L, van der Hulst R. Long-term Follow-up of Autologous Fat Transfer vs Conventional Breast Reconstruction and Association With Cancer Relapse in Patients With Breast Cancer. JAMA Surg 2018; in press.

16. Kronowitz SJ, Mandujano CC, Liu J, Kuerer HM, Smith B, Garvey P, et al. Lipofilling of the Breast Does Not Increase the Risk of Recurrence of Breast Cancer: A Matched Controlled Study. Plast Reconstr Surg 2016; 137(2):385-93.

17. Petit JY, Botteri E, Lohsiriwat V, Rietjens M, De Lorenzi F, Garusi C, et al. Locoregional recurrence risk after lipofilling in breast cancer patients. Ann Oncol 2012; 23(3):582-8.

18. Caviggioli F, Maione L, Forcellini D, Klinger F, Klinger M. Autologous fat graft in postmastectomy pain syndrome. Plast Reconstr Surg 2011; 128(2):349-52.

19. Serra-Renom JM, Muñoz-Olmo JL, Serra-Mestre JM. Fat grafting in postmastectomy breast reconstruction with expanders and prostheses in patients who have received radiotherapy: formation of new subcutaneous tissue. Plast Reconstr Surg 2010; 125(1):12-8.

20. Doren EL, Parikh RP, Laronga C, Hiro ME, Sun W, Lee MC, et al. Sequelae of fat grafting postmastectomy: an algorithm for management of fat necrosis. Eplasty 2012; 12:e53. 
21. Parikh RP, Doren EL, Mooney B, Sun WV, Laronga C, Smith PD. Differentiating fat necrosis from recurrent malignancy in fat-grafted breasts: an imaging classification system to guide management. Plast Reconstr Surg 2012; 130(4):761-72.

22. Silva-Vergara C, Fontdevila J, Weshahy O, Yuste M, Descarrega J, Grande L. Breast Cancer Recurrence Is not Increased With Lipofilling Reconstruction: A Case-Controlled Study. Ann Plast Surg 2017; 79(3):243-248.

23. Fisher B, Anderson S, Bryant J, Margolese RG, Deutsch M, Fisher ER, et al. Twenty-year follow-up of a randomized trial comparing total mastectomy, lumpectomy, and lumpectomy plus irradiation for the treatment of invasive breast cancer. $\quad \mathrm{N}$ Engl $\mathrm{J}$ Med 2002; 347(16):1233-41.

24. Veronesi U, Cascinelli N, Mariani L, Greco M, Saccozzi R, Luini A, et al. Twenty-year follow-up of a randomized study comparing breast-conserving surgery with radical mastectomy for early breast cancer. N Engl J Med 2002; 347(16):1227-32.

25. Morrow M, Harris JR, Schnitt SJ. Surgical margins in lumpectomy for breast cancer--bigger is not better. N Engl J Med 2012; 367(1):79-82.

26. Petit JY, Rietjens M, Botteri E, Rotmensz N, Bertolini F, Curigliano G, et al. Evaluation of fat grafting safety in patients with intraepithelial neoplasia: a matched-cohort study. Ann Oncol 2013; 24(6):1479-84.

27. Bielli A, Scioli MG, Gentile P, Agostinelli S, Tarquini C, Cervelli V, et al. Adult adiposederived stem cells and breast cancer: a controversial relationship. Springerplus 2014; 3:345.

28. Petit JY, Maisonneuve P, Rotmensz N, Bertolini F, Rietjens M. Fat Grafting after Invasive Breast Cancer: A Matched Case-Control Study. Plast Reconstr Surg 2017; 139(6):1292-1296.

29. Perou CM, Sørlie T, Eisen MB, van de Rijn M, Jeffrey SS, Rees CA, et al. Molecular subtypes of human breast tumours. Nature 2000; 406(6797):747-52. 
30. Sørlie T, Perou CM, Tibshirani R, Aas T, Geisler S, Johnsen H, et al. Gene expression patterns of breast carcinomas distinguish tumor subclasses with clinical implications. Proc Natl Acad Sci U S A 2001; 98(19):10869-74.

31. Dent R, Trudeau M, Pritchard KI, Hanna WM, Kahn HK, Sawka CA, et al. Triplenegative breast cancer: clinical features and patterns of recurrence. Clin Cancer Res 2007; $13: 4429-34$.

32. van Maaren MC, de Munck L, Strobbe LJA, Sonke GS, Westenend PJ, Smidt ML, et al. Tenyear recurrence rates for breast cancer subtypes in the Netherlands: A large population-based study. Int J Cancer 2019; 144(2):263-272.

33. Khan LR, Raine CR, Dixon JM. Immediate lipofilling in breast conserving surgery. Eur J Surg Oncol 2017; 43(8):1402-1408.

\section{Tables}

Table 1. Distribution of baseline variables after exact matching

\begin{tabular}{lccc}
\hline & $\begin{array}{c}\text { AFT } \\
(\mathbf{n = 2 3 3 )}\end{array}$ & $\begin{array}{c}\text { No AFT } \\
(\mathbf{n = 5 9 7})\end{array}$ & p-value \\
\hline Age at diagnosis (years) & $49.4( \pm 9.0)$ & $50.7( \pm 8.9)$ & 0.07 \\
Body mass index (mean, $\left.\mathbf{K g} / \mathbf{m}^{\mathbf{2}}\right)$ & $23.8( \pm 4.8)$ & $24.5( \pm 4.9)$ & 0.09 \\
Menopausal status & & &
\end{tabular}


Pre-menopausal

Post-menopausal

$134(57.5 \%)$

$99(42.5 \%)$

Type of surgery

Lumpectomy

$54(23.2 \%)$

Mastectomy

$179(76.8 \%)$

$26(11.2 \%)$

$173(74.2 \%)$

$34(14.6 \%)$

$23(9.9 \%)$

$126(54.1 \%)$

$84(36.0 \%)$

$31(13.3 \%)$

$94(40.3 \%)$

$71(30.5 \%)$

$37(15.9 \%)$

$120(51.5 \%)$

$75(32.2 \%)$

$16(6.9 \%)$

$22(9.4 \%)$

$186(79.8 \%)$

$47(20.2 \%)$

$107(45.9 \%)$

$28(12.0 \%)$

$98(42.1 \%)$

Yes, adjuvant

\section{Radiation therapy}

Yes

No
$342(57.3 \%)$

0.95

$255(42.7 \%)$

$444(74.4 \%)$

$153(25.6 \%)$

$<0.0001$

$62(10.4 \%)$

$455(76.2 \%)$

0.84

$80(13.4 \%)$

$72(12.1 \%)$

$345(57.8 \%)$

0.23

$180(30.1 \%)$

$72(12.1 \%)$

$289(48.4 \%)$

$178(29.8 \%)$

0.05

$58(9.7 \%)$

$336(58.3 \%)$

$181(30.3 \%)$

$28(4.7 \%)$

$52(8.7 \%)$

0.48

$475(79.6 \%)$

$122(20.4 \%)$

$331(55.4 \%)$

$50(8.4 \%)$

0.04

$216(36.2 \%)$

447 (74.9\%)

$150(25.1 \%)$ 
Table 2. Long-term outcomes between lipofilled vs. non-lipofilled patients

\begin{tabular}{lccc}
\hline & $\begin{array}{c}\text { AFT } \\
(\mathbf{n = ~ 2 3 3 )}\end{array}$ & $\begin{array}{c}\text { No AFT } \\
(\mathbf{n}=\mathbf{5 9 7})\end{array}$ & p-value \\
\hline Loco-regional recurrence & & & \\
Yes & $15(6.4 \%)$ & $30(5.0 \%)$ & 0.42 \\
No & $218(93.6 \%)$ & $567(95.0 \%)$ &
\end{tabular}


Distant metastases

\begin{tabular}{lccc} 
Yes & $18(7.7 \%)$ & $32(5.4 \%)$ & 0.20 \\
No & $215(92.3 \%)$ & $565(94.6 \%)$ & \\
Cancer-related death & $12(5.1 \%)$ & $20(3.4 \%)$ & 0.23 \\
Yes & $221(94.9 \%)$ & $577(96.6 \%)$ & \\
\hline No & & & \\
\hline
\end{tabular}

Table 3. Multivariate survival analysis of AFT vs. no AFT on 10-year LRR-free and DM-free survival probabilities

\begin{tabular}{lcccccc}
\hline & \multicolumn{2}{c}{ Loco-regional recurrence } & \multicolumn{2}{c}{ Distant Metastases } \\
\hline & $\begin{array}{c}\text { Hazard } \\
\text { Ratio }\end{array}$ & $\mathbf{9 5 \%}$ CI & p-value & $\begin{array}{c}\text { Hazard } \\
\text { Ratio }\end{array}$ & 95\% CI & p-value \\
\hline Age at diagnosis & 1.03 & $(0.98-1.08)$ & 0.29 & 1.02 & $(0.98-1.07)$ & 0.35 \\
Body mass index & 0.95 & $(0.88-1.03)$ & 0.19 & 0.93 & $(0.86-1.00)$ & 0.05
\end{tabular}




\section{Menopausal status}

Pre-menopausal vs. post-

menopausal

1.58

(0.66-3.79)

0.31

1.35

(0.58-3.12)

0.49

\section{Type of surgery}

Lumpectomy vs. mastectomy

0.84

(0.34-2.10)

0.71

1.09

(0.47-2.51)

0.84

\section{AFT}

Performed vs. not performed

0.87

(0.43-1.76)

0.69

0.82

(0.43-1.57)

0.55

pT stage

Early vs. locally-advanced breast cancer

\section{pN stage}

pN0 vs. pN2-3

$\begin{array}{llllll}1.14 & (0.35-3.76) & 0.83 & 0.42 & (0.16-1.07) & 0.07 \\ 1.45 & (0.45-4.67) & 0.54 & 0.54 & (0.23-1.30) & 0.17\end{array}$

pN1 vs. pN2-3

$\begin{array}{llllll}0.63 & (0.16-2.54) & 0.51 & 0.47 & (0.10-2.25) & 0.34 \\ 1.00 & (0.27-3.78) & 1.000 & 0.41 & (0.09-1.92) & 0.26 \\ 1.54 & (0.46-5.22) & 0.49 & 1.27 & (0.34-4.71) & 0.72\end{array}$

HER2+ vs. TNBC

Yes: neoadjuvant vs. no

\section{Hormone therapy}

Performed vs. not performed

0.70

(0.24-2.04)

0.51

2.41

(0.58-9.97)

0.22

\section{Radiation therapy}

Performed vs. not performed

1.04

(0.45-2.42)

0.93

0.71

(0.31-1.60)

0.40

Table 4. Impact of number of AFT procedures, time between oncologic surgery and AFT and type of surgery on LRR and DM

\begin{tabular}{lcccccc}
\hline $\begin{array}{l}\text { All AFT patients } \\
(\mathrm{n}=464)\end{array}$ & \multicolumn{2}{c}{ Loco-regional recurrence } & \multicolumn{3}{c}{ Distant metastases } \\
\hline & $\begin{array}{c}\text { No } \\
(n=439)\end{array}$ & $\begin{array}{c}\text { Yes } \\
(n=25)\end{array}$ & $\begin{array}{c}\text { Log-rank } \\
\text { p-value }\end{array}$ & $\begin{array}{c}\text { No } \\
(n=425)\end{array}$ & $\begin{array}{c}\text { Yes } \\
(n=39)\end{array}$ & $\begin{array}{c}\text { Log-rank } \\
\text { p-value }\end{array}$ \\
\hline
\end{tabular}

Number of AFT

procedures 
1

2

$\geq 3$

Time from cancer surgery to first AFT

$<1$ year

1-3 years

$>3$ years

\section{Type of surgery}

\begin{tabular}{|c|c|c|c|c|c|}
\hline Lumpectomy & $116(26.4 \%)$ & $5(20.0 \%)$ & 0.85 & $115(27.1 \%)$ & $6(15.4 \%)$ \\
\hline Skin-sparing mastectomy & $254(57.9 \%)$ & $15(60.0 \%)$ & & $243(57.2 \%)$ & $26(66.7 \%)$ \\
\hline Nipple-sparing mastectomy & $69(15.7 \%)$ & $5(20.0 \%)$ & & $67(15.8 \%)$ & $7(17.9 \%)$ \\
\hline
\end{tabular}

$\begin{array}{llllll}165(37.6 \%) & 5(20.0 \%) & 0.28 & 159(37.4 \%) & 11(28.2 \%) & 0.45 \\ 213(48.5 \%) & 16(64.0 \%) & & 206(48.5 \%) & 23(59.0 \%) & \\ 61(13.9 \%) & 4(16.0 \%) & 60(14.1 \%) & 5(12.8 \%)\end{array}$

$106(24.2 \%) \quad 9(36.0 \%)$

0.44

$107(25.2 \%)$

$8(20.5 \%)$

0.74

$198(45.1 \%) \quad 11(44.0 \%)$

$190(44.7 \%)$

$19(48.7 \%)$

$135(30.7 \%) \quad 5(20.0 \%)$

$128(30.1 \%) \quad 12(30.8 \%)$

$67(15.8 \%) \quad 7(17.9 \%)$

\section{Figure captions}

Figure 1 Survival probability curves of AFT vs. control patients. a) 10-year loco-regional recurrencefree survival probability; b) 10-year distant metastases-free survival probability. AFT, autologous fat transfer. 
Figure 2 10-year survival probability curves of AFT vs. control patients for each biomolecular subtype of breast cancer. a) loco-regional recurrence and b) distant metastases in Luminal A; c) locoregional recurrence and d) distant metastases in Luminal B; e) loco-regional recurrence and f) distant metastases in HER2-positive; g) loco-regional recurrence and h) distant metastases in triple-negative breast cancer. 